
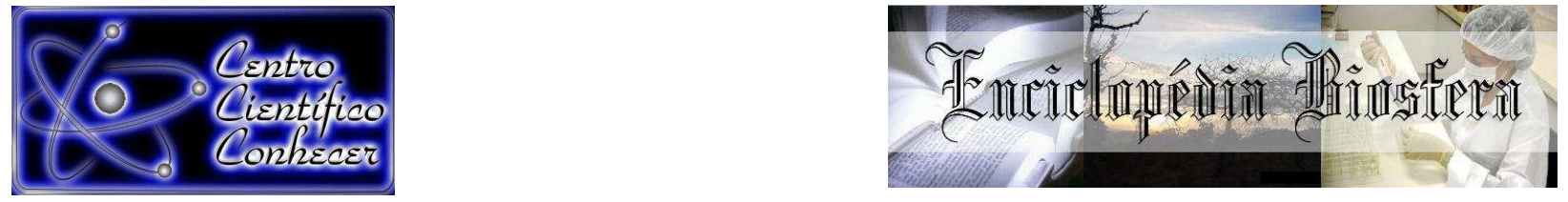

\title{
CARACTERÍSTICAS FÍSICO-QUÍMICAS DE ÓLEOS ESSENCIAIS DE PLANTAS DA REGIÃO DO VALE DO JURUÁ
}

\author{
William Ferreira Alves ${ }^{1}$, Maria Cristina de Souza ${ }^{2}$, Alda Naíne Silva Almeida ${ }^{3}$ Sabrina \\ Silva de Oliveira ${ }^{4}$, Ingrid Loraine Rocha Ribeiro ${ }^{5}$, \\ ${ }^{1}$ Professor Doutor da Universidade Federal do Acre, Cruzeiro do Sul, \\ (wfa23@yahoo.com.br) Acre, Brasil \\ ${ }^{2}$ Professora Doutora da Universidade Federal do Acre, Cruzeiro do Sul, Acre, Brasil \\ ${ }^{3}$ Graduada em Engenharia Agronômica da Universidade Federal do Acre, Cruzeiro \\ do Sul, Acre, Brasil. \\ ${ }^{4}$ Graduando em Engenharia Florestal da Universidade Federal do Acre, Cruzeiro do \\ Sul, Acre, Brasil \\ ${ }^{5}$ Graduando em Engenharia Florestal da Universidade Federal do Acre, Cruzeiro do \\ Sul, Acre, Brasil
}
Recebido em: 08/09/2015 - Aprovado em: 14/11/2015 - Publicado em: 01/12/2015 DOI: http://dx.doi.org/10.18677/Enciclopedia_Biosfera_2015_107

\begin{abstract}
RESUMO
Neste trabalho foram analisados experimentalmente os parâmetros físico e químico dos óleos essenciais de frutos de "coco" (Cocos nucifera L.),"açaî" (Euterpe oleracea Mart.), "patoá" (Oenocarpus bataua Mart.), "andiroba" (Carapa guianensis Aubl.), "copaíba" (Copaifera spp,),"buriti" (Mauritia flexuosa L.f.); e as manteigas de "murmuru" (Astrocaryum murumuru Mart.), "tucumã" (Astrocaryum vulgare Mart.) e "cupuaçu" (Theobroma grandiflorum (Willd.ex Spreng.) K.Schum.) As amostras foram provenientes do município de Mâncio Lima, Estado do Acre. Foram determinados os valores de índice de acidez, índice de refração, análise de espectrometria e determinação da viscosidade de acordo com as normas do Instituto Adolfo Lutz. Os índices de acidez foram de 0,701 $\pm 0,008$ e 6,69 $\pm 0,13$ para óleo de buriti e óleo de copaíba, respetivamente. $O$ índice de refração apontou valores de 1,4565 para o óleo de "coco" e manteiga de "tucumã", e 1,5010 para o óleo de copaíba. Os valores de massa molar média foram menores para as manteigas de "murmuru" e "tucumã", de acordo com os resultados de índice de saponificação. Os espectros de UV-VIS dos óleos essenciais apresentaram bandas distintas na região de $200 \mathrm{~nm}$ a $600 \mathrm{~nm}$.
\end{abstract}

PALAVRAS-CHAVE: Óleos essenciais; parâmetros físico químico, UV-VIS

\section{PHYSICOCHEMICAL CHARACTERISTICS OF THE ESSENTIAL OILS OF PLANTS IN THE REGION OF VALE DO JURUA}

\section{ABSTRACT}

In this work were experimentally analyzed the physical and chemical parameters of essential oils of fruits of "coconut" (Cocos nucifera L.), "açaí" (Euterpe oleracea Mart.), "patoá" (Oenocarpus bataua Mart.), "andiroba" (Carapa guianensis Aubl.), "copaiba" (Copaifera spp.), "buriti" (Mauritia flexuosa L.f.); and the butters of "murmuru" (Astrocaryum murumuru Mart.), "tucumã" (Astrocaryum vulgare Mart.) and ENCICLOPÉDIA BIOSFERA, Centro Científico Conhecer - Goiânia, v. 11 n.22; p.534 2015 
"cupuaçu" (Theobroma grandiflorum (Willd.ex Spreng.) K.Schum.). The samples were from the city of Mâncio Lima, state of Acre, being determined, viscosity, values of acidity, refraction, saponification and peroxide, and spectrophotometry analyses, accordingly to Adolfo Lutz Institute regulations. The acidity rates were 0,701 $\pm 0,008$ and 6,69 $\pm 0,13$ for buriti oil and copaiba oil, respectively. The refraction rates presentefd values of 1.4565 for "coconut" oil and "tucumã" butter, and 1.5010 for copaiba oil. The values of average molar mass were smaller for "murmuru" and "tucumã" butter, according to the results of saponification rates. The spectra of UVVIS of essential oils presented distinct bands in the region from $200 \mathrm{~nm}$ to $600 \mathrm{~nm}$.

KEYWORDS: physical chemical parameters, UV-VIS, essential oils

\section{INTRODUÇÃO}

Um dos biomas de grande importância mundial, a Amazônia apresenta uma biodiversidade surpreendente. No Brasil, tem-se gerado uma atenção especial a floresta amazônica devido a exploração irracional dos seus recursos florestais (PINTO et al. 2002).

Segundo a SUFRAMA, "a Amazônia Ocidental detém 42,97\% da extensão territorial da Amazônia Legal e comporta aproximadamente 57\% das florestas da região" e caracteriza-se por dois grandes tipos de ecossistemas: as áreas de terras de várzeas e as florestas de terra firme (SCHERER, 2004), ambas de fundamental importância dentro da dinâmica populacional das árvores nativas (SANTANA, 2015).

A Amazônia apresenta uma ampla diversidade de espécies florestais com grande potencial à produção de óleos e resinas usados por povos nativos e populações tradicionais para os mais variados fins. No Acre, a base econômica do estado baseia-se no extrativismo, entre os produtos de grande relevância econômica destacam-se o cupuaçu, o açaí, o buriti, a castanha-do-brasil (SUFRAMA, 2015).

A busca demasiada por produtos que atendam a demanda das indústrias de cosméticos e fitoterápicos tem gerado a exploração e a busca por óleos vegetais diferenciados. Os óleos essenciais extraídos apresentam ricas propriedades em sua composição química e uma variedade de aplicações no mercado, como cosméticos, fito-cosméticos, sabonetes, dentre outras. Diante de tamanha riqueza oferecida pela natureza, os óleos essenciais representam um grande potencial econômico para a Amazônia, pois traz alternativas de diversificação de produção e renda complementar para as famílias extrativistas (SOUZA et al., 2010).

Os óleos vegetais extraídos de folhas, cascas ou sementes são misturas complexas de substâncias voláteis (aroma e sabores característicos). Na temperatura ambiente apresentam-se como líquidos oleosos de alta volatilidade, na maioria dos casos, incolores ou ligeiramente amarelados. Alguns dos óleos vegetais, entretanto, têm outras cores, como é o caso do óleo volátil de camomila, que é azulado em decorrência de seu alto teor de azuleno; sendo que a maioria dos óleos vegetais é instável, na presença de luz, calor e umidade (SIMÕES et al., 1999).

Uma das maneiras de obter óleo essencial das folhas, cascas e semente, é por arraste de vapor produzido pelo processo de ebulição da água contendo o material botânico intacto ou extração por prensagem. Por arraste a vapor, o material volátil é arrastado pelo vapor d'água e posteriormente separado por decantação, e por prensagem, as folhas, cascas ou sementes, são prensadas até a saída do óleo vegetal (BRUNETON, 1991).

O aumento nas publicações de trabalhos relacionados a óleo essencial duplica aproximadamente a cada quatro anos, de acordo com os dados da Web of Science com as palavras chaves em inglês, "essential oils", "antioxidant" e 
"inflammatory" entre 2004 a 2014. Em 2004, em torno de 30 publicações foram catalogadas, enquanto que em 2014 foram publicados 209 trabalhos. Um fato importante é o aparecimento de trabalhos com óleos essenciais em várias revistas de alcance multidisciplinar, envolvendo áreas como química, física, biologia, medicina, matemáticas e engenharia. Tendo com isso integração de vários pesquisadores de várias áreas de conhecimento. Das 100 revistas que apresentam em suas publicações, referente a palavras chaves "essential oils", "antioxidant" e "inflammatory", são Microchemical Jornal, Acta Amazonica, Pharmacology Pharmacy, Biochemistry Molecular Biology, Chemistry, Immunology, Jornal of Technology Managemet \& Innovation, Brazilian Journal Vet. Res. Animal Science, Ciência e tecnologia de Alimentos, Clinical Research, Grasas y aceites, Química Nova, Food Chemistry, Journal of Medical Food, Food Research International, Journal of Functional Foods etc (WEBOFSCIENCE, 2015).

O Açaí ou Açaizeiro, espécie monocotiledônea, é uma palmeira nativa da região Amazônica, distribuído em cinco países. No Brasil, especificamente, o açaizeiro se encontra nos Estados do Amazonas, Amapá, Pará, Maranhão, Rondônia, Acre e Tocantins (YUYAMA et al., 2011; MARTINEZ, et al. 2013; VILAPLANA et al., 2014).

Segundo TINOCO (2005), do fruto do Açaí pode ser aproveitado praticamente tudo. Por exemplo, em torno de $80 \%$ do peso do Açaí é utilizado na produção de cosméticos, placas acústicas, óleo comestível entre outros, como produção de calor para torrefação de café, panificação, carvão vegetal e adubo orgânico. O açaí possui alto valor energético, podendo atingir ate $250 \mathrm{kcal}$ por $100 \mathrm{~g}$, muito rico em proteínas, vitaminas e gordura, principalmente monoinsaturadas e poli-insaturadas que, são benéficas e auxiliam na redução do colesterol ruim, também possui propriedades sensoriais únicas e grande potencial benéfico a saúde (EMBRAPA, 2014)

A Andiroba (Carapa guianensis) é uma árvore de grande porte pertencente à família Meliaceae, podendo atingir até $55 \mathrm{~m}$ de altura (FERRAZ et al., 2002), sementes apresentam coloração marrom e geralmente são grandes (ORELLANA et al., 2004), que se distribui por todo norte da América do Sul, incluindo a região Amazônica. É encontrada, predominantemente, em florestas de várzeas e área alagadiças (MARTINS et al., 2012; CAMPOS et al., 2013).

O óleo extraído das sementes da Andiroba, ao longo da história do Amazonas, teve uma importante participação na economia regional e continua sendo muito apreciado, em especial na medicina popular, devido as suas propriedades químicas. A coleta das sementes é de baixo custo, além de ser uma prática não destrutiva, a produção do óleo pode garantir um retorno econômico para a comunidade local (MENDONÇA \& FERRAZ, 2007; CAMPOS et al. 2013).

O Tucumã (Astrocaryum aculeatum) é uma palmeira encontrada nos estados do Acre, Amazonas, Pará e Rondônia. Cresce nas margens dos rios, em áreas não abertas com água e terra firme. Os seus frutos são utilizados na alimentação humana e animal. São elipsoides, amarelos ou alaranjados com tons avermelhados. Dele é possível extrair um óleo que na sua composição apresenta grande quantidade do precursor da vitamina $A$, superando em seis vezes a cenoura. Possui $74,4 \%$ e $25,6 \%$ de ácidos graxos insaturados e saturados, respectivamente, que são ricos em ácidos graxos, ômega 3, 6 e 9 (NAZÁRIO \& FERREIRA, 2010; SOUZA FILHO et al., 2013).

O Buriti (Mauritia flexuosa) representa uma das maiores palmeiras da floresta amazônica. Devido ao fato de poder ser empregado em diversas áreas, contribui para o aumento da capacidade econômica da região. Predomina em florestas de 
várzeas, sendo facilmente encontrado às margens de rios, lagos, lagoas, açudes etc. Esta palmeira é de amplo aproveitamento, desde a folha até a raiz. Da polpa dos frutos é possível produzir doces, vinhos; e do creme do fruto é extraído o óleo, com empregos estético, terapêutico e atividade antioxidante, ressaltando que o mesmo possuí alto teor de vitamina $A$. Das folhas e da madeira são fabricados produtos artesanais (LORENZI et al., 2004; CARVALHO et al., 2011; CÂNDIDO et al., 2015).

As copaíbas (Copaifera spp) são de ampla ocorrência, sendo encontradas desde o México ao norte da Argentina, e na África Ocidental. São árvores da família Fabaceae-Caesalpinoideae, que podem atingir até 40 metros de altura. Apresentam uma madeira de boa qualidade e no interior de seu tronco existe uma produção de óleo-resina, que em termos biológicos é um produto de desintoxicação que funciona como defesa contra fungos, animais e bactérias. O óleo-resina de copaíba é muito utilizado pelas populações tradicionais e indústrias farmacêuticas devido às suas propriedades terapêuticas, como cicatrizante e anti-inflamatório (LEANDRO et al., 2012; NOGUEIRA et al., 2012).

O Patoá (Oenocarpus bataua) apresenta grande quantidade de proteínas, podendo seu teor ser comparado com a carne e o leite de gado (FIGUEIRA, 2012). Pertence à família botânica Arecaceae, com preferência por lugares úmidos. No Brasil, ocorre nos Estados do Acre, Amazonas, Pará, Rondônia e até uma parte da Região Centro-Oeste (LORENZI et al., 2004; RODRIGUES et al. 2010). Só produz a partir do oitavo ano, podendo demorar entre 10 a 14 meses para se desenvolver. Da polpa do fruto, extrai-se o óleo que é muito utilizado na alimentação, consistindo de um liquido amarelo esverdeado ou transparente, semelhante ao óleo de oliva (DARNET et al., 2011; AMAZON OIL, 2015).

A palmeira Murmuru (Astrocaryum ulei) é abundante na região amazônica brasileira estendendo-se até a fronteira com a Bolívia e Peru. Seu fruto tem coloração avermelhada quando maduro com uma polpa amarela e uma amêndoa rica em óleo, de onde é extraído o ingrediente principal, muito utilizado na indústria de cosméticos para o tratamento dos cabelos e pele. Outra característica própria e de destaque dessa palmeira é o fato de seus cachos, repletos de cocos, crescerem voltados para cima (LORENZI et al., 2004; NASCIMENTO, 2007; ABREU et al., 2014).

O coco (Cocos nucifera), palmeira exótica, de fácil adaptação e cultivo na maioria dos continentes, mostra-se como produto valioso na indústria in natura, pois a água proveniente dele é um isotônico natural, estando presente na cavidade da semente, rica em nutrientes, possuindo $5 \%$ de açúcares, além de proteínas, vitaminas e sais minerais, sendo uma bebida leve, refrescante e pouco calórica (MARTINS \& JESUS JUNIOR, 2011). O óleo, possui grandes propriedades medicinais, cujos estudos recentes apontam atividades tais como anti-inflamatório, analgésico e antipirético (INTAHPHUAK et al., 2014)

Considerando o fato que na produção extrativista, desenvolvida na maioria das regiões amazônicas, não há qualquer preocupação com as características físico-químicas dos óleos essenciais e nenhum dado experimental que acompanhe a extração dos óleos essenciais na sua safra, neste presente trabalho, o objetivo foi de analisar os parâmetros físicos químicos dos óleos essenciais da região do Vale do Juruá no extremo ocidental do Brasil. 


\section{MATERIAL E MÉTODOS}

\section{Identificação das espécies}

O material botânico foi identificado com base em caracterização morfológica e literatura especializada (LORENZI et al., 2004, LORENZI, 2010; OLIVEIRA \& SOUZA, 2014). Os nomes científicos foram revisados mediante consulta ao site do Jardim Botânico de Missouri (www.mobot.org).

Os óleos essenciais extraídos foram armazenados em um frasco fechado e conservados em uma geladeira comum para evitar a oxidação na presença do oxigênio e degradação por radiação da luz. Uma vez obtidas as amostras, procedeuse à verificação da qualidade das mesmas utilizando a análise do índice de acidez e densidade para todos os óleos.

Os experimentos foram desenvolvidos no Laboratório de Química e Solos da Universidade Federal do Acre - UFAC/Campus Floresta. E as analises de Espectroscopia na Região do Ultravioleta e no Visível (UV-vis) dos óleos essenciais foram realizadas no Laboratório de Polímeros da Universidade Estadual Paulista UNESP de Ilha Solteira - SP.

\section{Determinação da viscosidade}

A viscosidade de um fluído pode ser determinada por vários métodos: através da resistência de líquidos ao escoamento, tempo de vazão de um líquido (aproximadamente $10 \mathrm{ml}$ ) através de um capilar (viscosímetro de Oswald); da medida do tempo de queda de uma esfera através de um líquido (Höppler); medindo a resistência ao movimento de rotação de eixos metálicos quando imersos na amostra (50 $\mathrm{ml}$ do óleo). O método que foi utilizado neste trabalho foi o viscosímetro de rotação de eixos.

\section{Determinação da densidade}

A densidade do líquido pode ser determinada pela diferença de massa do liquido pelo volume ocupado. Um dos métodos rápido e fácil de determinar a densidade de liquido é o método do picnômetro. Por este método é possível determinar com exatidão e precisão a densidade de diversos líquidos em relação à densidade da água pura, sempre com auxílio de uma balança analítica. A quantidade de amostra será de $50 \mathrm{ml}$, volume total do picnômetro. A densidade dos óleos vegetais foi realizada em triplicata, com o valor de densidade média e seu respectivo desvio padrão $(\sigma)(I A L, 1985)$.

\section{Determinação do índice de refração}

O índice de refração é característico para cada tipo de óleo, dentro de certos limites. Está relacionado com o grau de saturação das ligações, mas é afetado por outros fatores tais como: teor de ácidos graxos livres, oxidação e tratamento térmico. Este método é aplicável a todos os óleos normais e gorduras líquidas. O índice de refração dos óleos vegetais foi determinado pelo polarímetro de disco, sendo realizado em triplicata, com o valor de refração média e seu respectivo desvio

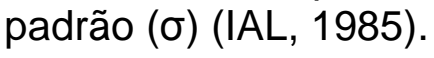

\section{Determinação da Acidez}

O índice de acidez é definido como o número de miligramas de hidróxido de potássio necessário para neutralizar um grama da amostra. O método é aplicável a óleos brutos e refinados, vegetais e animais, e gorduras animais. Os métodos que 
avaliam a acidez titulável resumem-se em titular com soluções de álcali-padrão, a acidez do produto ou soluções aquosas/alcoólicas do produto, assim como os ácidos graxos obtidos dos lipídios (IAL, 1985).

\section{Determinação da extinção específica por absorção na região do ultravioleta}

A análise espectrofotométrica na região do ultravioleta pode fornecer informações sobre a qualidade de um óleo, seu estado de conservação e alterações causadas pelo processamento. Neste método, o óleo essencial em questão é dissolvido em solvente apropriado (exemplo em uma relação de 10/0,3 de tolueno e óleo essencial $v / v$ ) e a extinção da solução é determinada nos comprimentos de onda especificados, usando como referência o solvente puro. Estas absorções são expressas como extinções específicas (IAL, 1985).

\section{Determinação do índice de saponificação}

Refere-se a quantidade de álcali necessária para saponificar uma quantidade definida de amostra. O método expressa o número de miligramas de hidróxido de potássio necessário para saponificar um grama de amostra (IAL, 1985).

\section{Determinação do índice de peróxido}

Esse índice determina, em termos de miliequivalentes de peróxido por $1000 \mathrm{~g}$ de amostra, que oxidam o iodeto de potássio nas condições do teste. Essas substâncias são geralmente consideradas como peróxidos ou outros produtos similares resultantes da oxidação da gordura (IAL, 1985).

\section{RESULTADOS E DISCUSSÃO}

Conforme se pode verificar na Tabela 1, foram observados, pela análise de índice de acidez, diferenças nos óleos essenciais de copaíba, andiroba e patoá, que apresentaram 6,93 $\pm 0,13 \mathrm{mg} / \mathrm{mL}$ de $\mathrm{NaOH}, 3,45 \pm 0,05 \mathrm{mg} / \mathrm{mL}$ de $\mathrm{NaOH}$ e 0,252 \pm $0,016 \mathrm{mg} / \mathrm{mL}$ de $\mathrm{NaOH}$, respectivamente. O alto valor de índice de acidez do óleo de copaíba pode estar relacionado ao tempo de armazenamento, após a extração do óleo. Isto é, à medida que o óleo mantido é exposto à radiação, as cadeias carbônicas das moléculas dos óleos essenciais se quebram. A ANVISA (2005) estabelece que valores de índice de acidez para óleos vegetais e manteigas vegetais maiores que $4,0 \mathrm{mg} / \mathrm{mL}$ de $\mathrm{NaOH}$ não são aceitáveis para consumo humano. Enquanto que o óleo de açaí, que apresentou menor valor de degradação, e os demais óleos, apresentaram valores de índice de acidez abaixo do índice de acidez do óleo de copaíba e andiroba (Tabela 1).

TABELA 1: Resultado das analise de índice de acidez e de densidade dos os óleos essenciais.

\begin{tabular}{ccc}
\hline Óleo & Índice de Acidez & Densidade $\mathbf{( m g} / \mathbf{m L})$ \\
\hline Copaíba & $6,93 \pm 0,13$ & $0,94 \pm 0,06$ \\
Buriti & $0,701 \pm 0,008$ & $0,91 \pm 0,03$ \\
Andiroba & $3,45 \pm 0,05$ & $0,87 \pm 0,02$ \\
Patoá & $0,252 \pm 0,016$ & $0,96 \pm 0,02$ \\
Açaí & $0,420 \pm 0,007$ & $0,82 \pm 0,01$ \\
Coco & $3,09 \pm 0,02$ & $0,86 \pm 0,02$ \\
Tucumã & $1,37 \pm 0,02$ & $0,85 \pm 0,02$ \\
Cupuaçu & $1,68 \pm 0,06$ & $0,87\left(40^{\circ} \mathrm{C}\right) \pm 0,02$ \\
Murmuru & $0,281 \pm 0,008$ & $0,91\left(40^{\circ} \mathrm{C}\right) \pm 0,02$ \\
\hline
\end{tabular}

Fonte: Próprio autor

ANGELUCCI et al. (1987) observaram que o alto teor de acidez de um óleo bruto aumenta a perda da neutralização, sendo também indicador de sementes de baixas qualidades, de manuseio e armazenamento impróprios ou de um 
processamento insatisfatório. De maneira semelhante, RIBEIRO \& SERAVALLI (2004) revelam que o estado de conservação do óleo está intimamente relacionado com a natureza e qualidade da matéria-prima, com a qualidade e o grau de pureza do óleo, com o processamento e, principalmente, com as condições de conservação.

A densidade é determinada pela relação entre a massa de uma substância e seu volume, expressa em gramas por centímetros cúbicos. Os valores de densidade dos óleos apresentaram diferenças, 0,96 $\pm 0,06 \mathrm{mg} / \mathrm{mL}$ para o óleo de patoá e 0,82 $\pm 0,01 \mathrm{mg} / \mathrm{mL}$ para o óleo de açaí, indicando que esse último possui em sua cadeia carbônica maior grau de insaturações (Tabela 1). De acordo com RIBEIRO \& SERAVALLI (2004), a medida de densidade do óleo está relacionada ao grau de ligações duplas ou triplas, ou seja, quanto menor for o peso molecular do óleo, maior é o grau de insaturações.

A Tabela 2 apresenta as viscosidades em mPa.s de cada óleo essencial, analisado por viscosímetro rotativo. Pode-se observar que o óleo de copaíba obteve maior valor de viscosidade. Entretanto, os óleos essenciais de murmuru e cupuaçu, na temperatura de $25{ }^{\circ} \mathrm{C}$, apresentam em seu estado físico solidificado. Com isso, foram realizados à temperatura de $40^{\circ} \mathrm{C}$.

Conforme MULLER (1978), óleos com alta viscosidade apresentam em sua estrutura molecular grupos hidroxilas, que explicam a formação de interações como pontes de hidrogênio, além de possuírem estabilidade oxidativa.

TABELA 2: Valores de viscosidade dos óleos essenciais a $25^{\circ} \mathrm{C}$.

\begin{tabular}{cc}
\hline Óleo essencial & Viscosidade (mPa.s) \\
\hline Copaíba & $45,0 \pm 0,2$ \\
Buriti & $15,0 \pm 0,5$ \\
Andiroba & $18,3 \pm 0,3$ \\
Patuá & $18,3 \pm 0,2$ \\
Açaí & $20,0 \pm 0,3$ \\
Coco & $12,7 \pm 0,3$ \\
Tucumã & $12,0\left(40^{\circ} \mathrm{C}\right) \pm 0,2$ \\
Murmuru & $10,0\left(40^{\circ} \mathrm{C}\right) \pm 0,1$ \\
Cupuaçu & $12,0\left(40^{\circ} \mathrm{C}\right) \pm 0,2$ \\
\hline
\end{tabular}

Fonte: Próprio autor

O índice de refração é característico para cada tipo de óleo, dentro de certos limites. Este relacionado com o grau de saturação das ligações, mas é afetado por outros fatores tais como: teor de ácidos graxos livres, oxidação e tratamento térmico. Este método é aplicável a todos os óleos normais e gorduras líquidas (IAL, 1985). $\mathrm{Na}$ Tabela 3, observa-se uma pequena diferença nos valores dos índices de refração de cada óleo analisado, em duas temperaturas diferentes, $20{ }^{\circ} \mathrm{C}$ e $40{ }^{\circ} \mathrm{C}$.

TABELA 3: Valores de índice de refração dos óleos analisados nas temperaturas de $20^{\circ} \mathrm{C}$ e $40^{\circ} \mathrm{C}$, de acordo com as normas do INSTITUTO ADOLFO LUTZ, 1985.

\begin{tabular}{ccc}
\hline $\begin{array}{c}\text { Tipo de Óleo Essencial e/ou } \\
\text { Manteiga }\end{array}$ & Temperatura $\mathbf{2 0}^{\circ} \mathbf{C}$ & Temperatura $\mathbf{4 0} \mathbf{0}^{\circ} \mathbf{C}$ \\
\hline Patoá & 1,4670 & 1,4635 \\
Buriti & 1,4665 & 1,4610 \\
Açaí & 1,4670 & 1,4620 \\
Tucumã & 1,4565 & 1,4510 \\
Coco & 1,4565 & 1,4500 \\
Andiroba & 1,4650 & 1,4605 \\
Copaíba & 1,5010 & 1,4950 \\
Cupuaçu & 1,4665 & 1,4575 \\
Murmurú & 1,4820 & 1,4500 \\
\hline
\end{tabular}

Fonte: Próprio autor 
Com relação ao índice de saponificação, observa-se na Tabela 4, que os óleos essenciais de andiroba, buriti, patoá e copaíba, apresentaram menores valores. Enquanto que os óleos de tucumã, murmuru, cupuaçu e coco (cozido e prensado) apresentaram maiores valores de índice de saponificação. Já com relação a massa molar média ¿os óleos essenciais de andiroba, buriti, patoá e copaíba apresentaram maiores valores de massa molar média, ou seja, possuem quantidades de moléculas com diferentes tamanhos e ou pesos moleculares.

TABELA 4: Valores de índice de saponificação e de peróxidos dos óleos essenciais.

\begin{tabular}{ccc}
\hline Óleo essencial & $\mathbf{m g ~ K O H} / \mathbf{g}$ de oleo & Meq/1000g de óleo \\
\hline Tucumã & $149,8 \pm 8,1$ & $22 \pm 7$ \\
Murmuru & $150,3 \pm 7,3$ & $34 \pm 3$ \\
Cupuaçu & $125,7 \pm 6,4$ & $28 \pm 5$ \\
Andiroba & $80,2 \pm 7,7$ & $18,6 \pm 4$ \\
Buriti & $101,0 \pm 6,1$ & $4 \pm 0,5$ \\
Patauá & $97,6 \pm 3,5$ & $8 \pm 2$ \\
Copaiba & $57,2 \pm 8,2$ & $154 \pm 7$ \\
Açai & $104,6 \pm 7,1$ & $1 \pm 0,02$ \\
Coco & $132,4 \pm 8,8$ & $26 \pm 4$ \\
\hline
\end{tabular}

Fonte: Próprio autor

De acordo com a literatura, a saponificação refere-se à reação do hidróxido alcalino ou alcalino terroso com a gordura (ésteres dos óleos essenciais) formando glicerol e sais alcalinos. O índice de saponificação se refere ao número que representa a massa (em miligramas) de hidróxido de potássio necessária para saponificar $1 \mathrm{~g}$ de óleo ou gordura (IAL, 1985). Este representa uma medida dos ácidos graxos livres e combinados que existem no óleo e é diretamente proporcional à massa molar média. Quanto maior o índice de saponificação menor é a massa molar média do óleo essencial (SCRIMGEOUR, 2005). Além disso, também, representa um importante parâmetro de grau de oxidação de gordura ou o óleo, sendo uma das principais formas de degradação e responsável pelo aparecimento de alguns odores estranhos, além do aumento de produtos tóxicos devido a degradação (MORETTO \& FETT, 1998).

Segundo CECCHI (2003), o índice de peróxido é um dos métodos mais utilizados para medir a oxidação dos óleos essenciais. A determinação do índice de peróxido consiste de determinar para todas as substâncias a quantidade de amostras que oxidam com o iodeto de potássio, em miliequivalentes de peróxido por $1000 \mathrm{~g}$ de amostras, devido à sua ação fortemente oxidante, os peróxidos orgânicos formados no início da rancificação, atuam sobre o iodo de potássio, liberando o iodo que será titulado com tiossulfato de sódio em presença de amido, como indicador (IAL, 1985; MORETTO \& FETT, 1998). Segundo MALACRIVA (2003), valores acima de $10 \mathrm{meq} / 1000 \mathrm{~g}$ de óleo indicam uma baixa degradação oxidativa.

Verificando-se ainda a Tabela 4 observa-se que o óleo de copaíba obteve maior valor de índice de peróxido, $154 \pm 15 \mathrm{meq} / 1000 \mathrm{~g}$., indicando para o óleo de copaíba alto grau de degradação, comparado com os óleos de murmuru, cupuaçu, coco e andiroba, que obtiveram valores em torno de 18 a $34 \mathrm{meq} / 1000 \mathrm{~g}$. Enquanto os óleos de açaí e buriti, apresentam valores abaixo de $10 \mathrm{meq} / 1000 \mathrm{~g}$.

Pela técnica de Uv-Vis foram observadas diferenças entre os espectros dos óleos essenciais estudados, principalmente para os óleos essenciais de açaí, tucumã, andiroba e copaíba como pode ser visto na Figura 1, sendo ainda observadas duas bandas de absorção, para o óleo essencial de açaí, nas regiões de ENCICLOPÉDIA BIOSFERA, Centro Científico Conhecer - Goiânia, v.11 n.22; p.541 2015 
350 a $500 \mathrm{~nm}$ e 650 a $700 \mathrm{~nm}$. Estas regiões compreendem a absorção da região do azul e do vermelho, respectivamente. Para o óleo essencial de tucumã, apresentase uma banda entre 400 a $550 \mathrm{~nm}$, que correspondem a absorção da região do azul. Enquanto que para os óleos essenciais de andiroba e copaíba, apresentaram uma forte absorção na região do ultravioleta (de $320 \mathrm{~nm}$ para valores mais baixo de comprimento de onda). Estes resultados demonstram diferença da estrutura da cadeia carbônica dos óleos observados, como, tipos de ligações, números de substâncias constituintes dos óleos, etc (RAVEN et al., 1996; GOEL, 1988; ALVES et al., 2014).

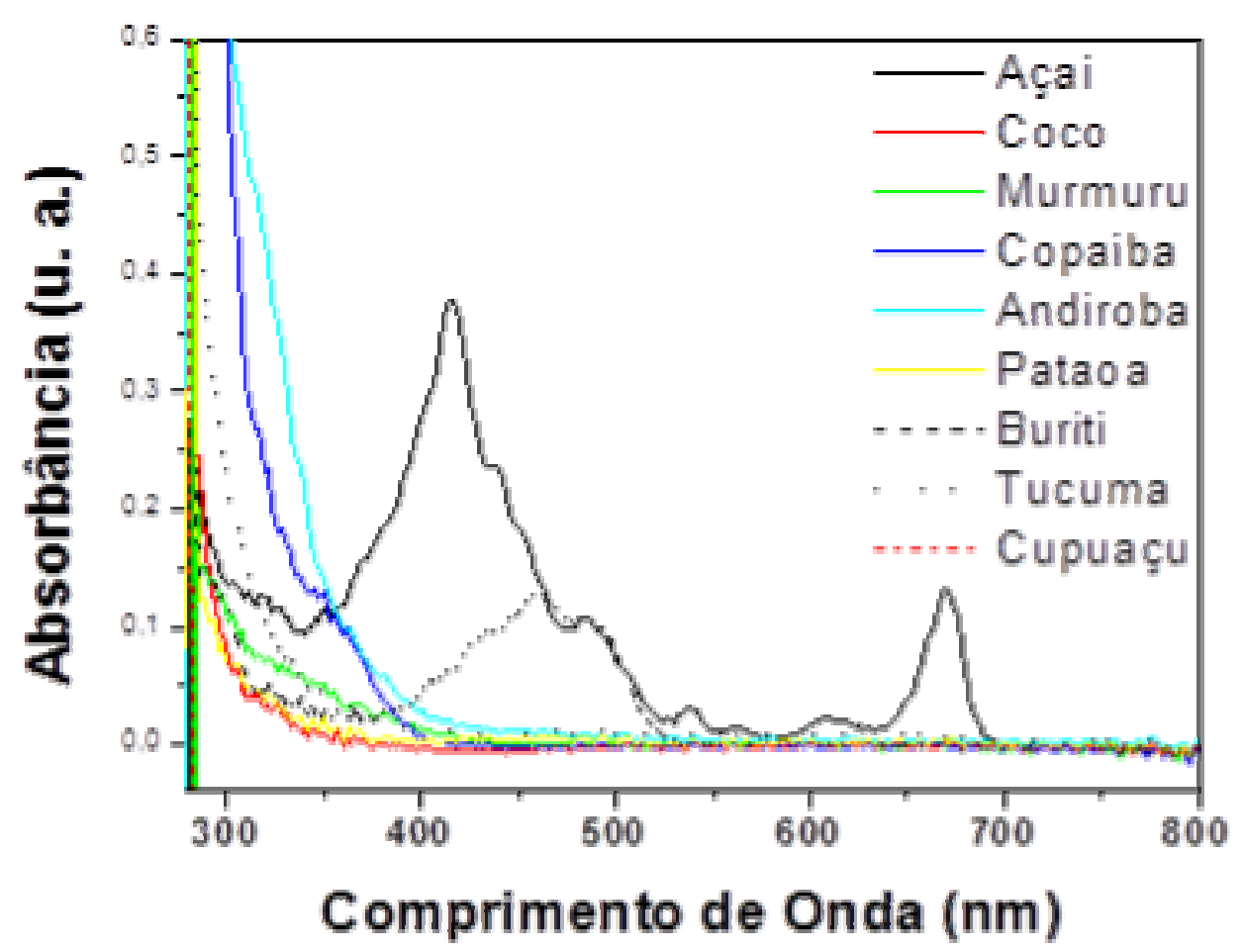

FIGURA 1. Espectros de absorção na região ultravioleta e visível dos óleos essenciais.

\section{CONCLUSÃO}

De acordo com os parâmetros físico-químicos apresentados, os óleos essenciais de buriti, andiroba, patoá, açaí, coco, e as manteigas de tucumã, cupuaçu e murmuru, analisados apresentaram-se com boa qualidade, nas análises de Índice de Acidez. Os valores de acidez estão relacionados à qualidade de conservação do óleo, quanto maior o valor de acidez, maior é seu estado de degradação. As análises dos parâmetros físicos químicos (Índice de Refração, Índice de Saponificação, Viscosidade, Densidade e Índice de Peróxido) apresentaram boa distinção dos óleos essenciais analisados. Os espectros de absorção UV-Vis indicaram bandas de absorção distintas entre os óleos, isto é, possuem diferentes "cores" que são devidos a diferentes substâncias encontradas nos óleos. Assim pode-se ressaltar que parcerias de cooperativas extrativistas com a Universidade Federal do Acre é fundamental para controle de qualidade e manejo florestal sustentável, de modo a se obter produtos com qualidade e melhoria de qualidade de vida das comunidades ribeirinhas do Vale do Juruá. 


\section{AGRADECIMENTOS}

Ao CNPq processo 474715/2011-6 e a FAPAC processo 024/2013 Edital 003/2013. Ao Prof. Dr. Jose Antônio Malmonge pelo uso dos equipamentos e auxílio nas análises dos dados.

\section{REFERÊNCIAS}

ABREU, M. G. P. de; FERREIRA, J. B; NEVES, Y. Y. B; ARAUJO, M. L. de; SOUZA, R. B. Efeito Fungitoxico de Óleos Essenciais de Palmeiras Amazônicas sobre Colletotrichum sp., Enciclopédia Biosfera, v.10, n.19, 2014.

ALVES, W. F.; ALMEIDA, A. N. S.; SOUZA, C. G.; ABDALLAH, D.O.; OLIVEIRA, S. $S$. de; Estudo comparativo dos espectros de FTIR e UV-VIS dos óleos essenciais da Amazônia Ocidental: In: Reunião Anual da SBPC, 66, 2014. Rio Branco. Anais... São Paulo, 2014.

AMAZON OIL, AMAZON OIL INDUSTRY, Pará, Brasil. Disponível em: <http://www.amazonoil.com.br/>. Acesso: 12 de junho de 2015.

ANVISA. Regulamento Técnico Para Óleos Vegetais, Gorduras Vegetais e Creme Vegetal. Diário Oficial da República Federativa do Brasil, Brasília, DF, 23 de set. 2005.

ANGELUCCI, E.; CARVALHO, L. R.; CARVALHO, N. R. P.; FIGUEIREDO, B. I.; MANTOVANI, B. M. D.; MORAES, M. R. Análise química de alimentos: Campinas, São Paulo, 1987. 123p. (Manual Técnico).

BRUNETON, J. Elementos de fitoquímica y de farmacognosia. Barcelona: editorial Acribiá, 1991.

CAMPOS, T., CUNHA, M. O., DE SOUSA, A. C. B., TEIXEIRA, R. B., RAPOSO, A., SEBBENN, A. M., WADT, L. H. O., Mating system parameters in a high density population of andirobas in the Amazon forest, Pesq. agropec. bras., Brasília, v.48, n.5, p.504-509, 2013.

CANDIDO, T. L. N, SILVA, M. R., AGOSTINI-COSTA, T. S., Bioactive compounds and antioxidant capacity of buriti (Mauritia flexuosa L.f.) from the Cerrado and Amazon biomes, Food Chemistry, v.177, n.15, p.313-319, 2015.

CARVALHO, C. OLIVEIRA; SCUDELLER, V. V.; SARGENTINI JUNIOR, É. ; FERNANDES, O. C. C. ;BOLSON, M. A. . Características físicas, químicas e rendimento do óleo de buriti (Maurita flexuosa L..f. Arecaceae). Diversidade Biológica. Manaus: editora Inpa, v.03, p.123-134, 2011

$\mathrm{CECCHI}, \mathrm{H}$. M. Fundamentos teóricos e práticos em análise de alimentos.

Editora da UNICAMP: $2^{\circ}$ Ed. rev.- Campinas, SP, editora da UNICAMP, 2003. 207p.

DARNET, S. H; SILVAI, L. H. M. da; RODRIGUES, A. M. da C; LINS, R. T. Nutritional composition, fatty acid and tocopherol contents of buriti (Mauritia flexuosa) and patawa (Oenocarpus bataua) fruit pulp from the amazon region. Ciênc. Tecnol. Aliment., Campinas, v.31, n.2, p.488-491, 2011 
EMBRAPA. Potencial de mercado de frutas da Amazônia. Disponível em: <http://www21.sede.embrapa.br/linhas_de_acao/alimentos/fruticultura/fruticultura_10 /mostra_documento>. Acesso: 11 de junho 2015.

FERRAZ, I. D. K.; CAMARGO, J. L. C.; SAMPAIO, P. T. B. Sementes e plântulas de andiroba (Carapa guianensis AUBL. E Carapa proera D. C.): aspectos botânicos, ecológicos e tecnológicos. Acta Amazônica, v32, n.4, p.647-661, 2002.

FIGUEIRA, L. C., Espectroscopia vibracional (no infravermelho e raman) e espectrofotometria de absorção uv-vis dos óleos de buriti (mauritia flexuosa) e de patauá (oenocarpus bataua). UNIVERSIDADE FEDERAL DO OESTE DO PARÁ, Santarém, Pará, Mar/2012, 57p.

NOGUEIRA ,E. de O; NOVAES, A. S. M.; SANCHEZ, C. M. S; ANDRADE, C. de M; SILVA, M. F. A. da. Avaliação do efeito do óleo-resina de copaíba (Copaifera sp.) na proliferação celular in vitro. Braz. J. Vet. Res. Anim. Sci., São Paulo, v.49, n.4, p.293-300, 2012

GOEL, N. S. Models of vegetation canopy reflectance and their use in estimation of biophysical parameters from reflectance data. Remote Sensing Reviews, .v.4, p.1$21,1988$.

INTAHPHUAK, S.; KHONSUNG, P.; PANTHONG, A.; Anti-inflammatory, analgesic, and antipyretic activities of virgin coconut oil. Pharmaceutical Biology, v.48, n.2, p.151-157, 2014.

INSTITUTO ADOLFO LUTZ. Normas analíticas do Instituto Adolfo Lutz. v.1.:Métodos Químicos e Físicos para Análise de Alimentos, 3. ed. São Paulo: IMESP, p.245-246. 1985.

LEANDRO, L. M.; VARGAS, F. S.; BARBOSA, P. C. S.; NEVES, J. K. O.; SILVA, J. A.; VEIGA-JUNIOR, V. F. Chemistry and biological activities of terpenoids from copaiba (Copaifera spp.) Oleoresins. Molecules, v.17, n.4, p.3866-3889, 2012.

LORENZI, H. Flora brasileira: Arecaceae (palmeiras). Nova Odessa, São Paulo: Instituto Plantarum, 2010, 368 p.

LORENZI, H.; NOBLICK, L; KAHN, F.; FERREIRA, E. Palmeiras Brasileiras e Exóticas Cultivadas. Nova Odessa, São Paulo: Instituto Plantarum, 416 p. 2004.

MALACRIDA, C. R. Alterações do óleo de soja e da mistura azeite de dendê - óleo de soja em frituras descontínuas de batatas chips. Braz. J. Food Technol. São Paulo, v.6, n.2, p. 245-249, 2003

MARTINS, C. R.; JESUS JÚNIOR, L. A. de; Evolução da produção de coco no Brasil e o comércio internacional - Panorama 2010; Embrapa Tabuleiros Costeiros, v.10, [s. n.], 2011 
MARTINS, K.; RAPOSO, A.; KLIMAS, C.A.; VEASEY, E.A.; KAINER, K.; WADT, L.H.O. Pollen and seed flow patterns of Carapa guianensis Aublet. (Meliaceae) in two types of Amazonian forest. Genetics and Molecular Biology, v.35, p.818-826, 2012.

MARTINEZ, E.J. L-; M.L.; CÓRDOVA F.; BARRALES; P. O.; MEDINA, A. R. Characterization and comparison of the chemical composition of exotic superfoods, Microchemical Journal, june, v. 110, [s. n.], p.444-451, 2013

MENDONÇA, A. P.; FERRAZ, I. D. K. Óleo de andiroba: processo tradicional na extração, uso e aspectos sociais no estado do Amazonas, Brasil. Acta Amazonica, v.37, n.3, p.353-364, 2007.

MORETTO, E.; FETT, R. Tecnologia de óleos e gorduras vegetais na indústria de alimentos. São Paulo: Varela, 1998.

MULLER, H.G. Introduccion a la reologia de los alimentos. Editora Acribia, Zaragoza, 1978. $174 \mathrm{p}$.

NASCIMENTO, Parâmetros Biométricos dos Cachos, Frutos e Sementesda Palmeira Murmuru (Astrocaryum ulei Burret.) encontrado na Região de Porto Acre. Revista Brasileira de Biociências, Porto Alegre, v.5, n.1, p.90-92, 2007.

NAZÁRIO, P; FERREIRA, S. A. do N. Emergência de plântulas de Astrocaryum aculeatum G. May. em função da temperatura e do período de embebição das sementes, Acta Amazonica, v.40, n.1, p. 165 - 170, 2010

OLIVEIRA, R.B.; SOUZA, M.C. Diversidade de Frutos da Região do Alto Juruá, Acre, Brasil. Enciclopédia Biosfera, v.10, n.19, p. 2129-2140, 2014.

ORELLANA, B. J. P; KOBAYASHI, E. S; LOURENÇO, G. M. Terapia alternativa através do uso da andiroba. Lato \& Sensu, v.5, n.1, p.136-141, 2004.

PINTO, A. A. M; SOUZA, A. L de; SOUZA, A. P de; MACHADO, C. C; MINETTE, L. J; VALE, A. B do. Análise de Danos de Colheita de Madeira em Floresta Tropical Úmida Sob Regime de Manejo Florestal Sustentado na Amazônia Ocidental. R. Árvore, Viçosa-MG, v.26, n.4, p.459-466, 2002.

RAVEN, P. H.; EVERT, R. F.; EICHHORN, S. E. Biologia vegetal. Rio de Janeiro: Guanabara Koogan, 1996. 728 p.

RODRIGUES, A. M. da C; DARNETB, S; SILVA, L. H. M. da; Fatty Acid Profiles and Tocopherol Contents of Buriti (Mauritia flexuosa), Patawa (Oenocarpus bataua), Tucuma (Astrocaryum vulgare), Mari (Poraqueiba paraensis) and Inaja (Maximiliana maripa) Fruits. J. Braz. Chem. Soc., v.21, n.10, p.2000-2004, 2010.

RIBEIRO, E. P.; SERAVALLI, E. A. G. Química de Alimentos, 2004. p.194. 
SANTANA, F. A.; Comunidades Ribeirinhas da Amazônia: Relato de Experiencia. Revista Perpectiva Amazonica. Disponivel em:www.fit.br/revista/doc/6 87.pdf. Acesso: 10 de agosto de 2015.

SCHERER, E. O defeso e a defesa do meio ambiente. Universidade Federal do Amazonas. 2004, 15p.

SCRIMGEOUR, C. Chemistry of Fatty Acids In: SHAHIDI, F. (Org.) Bailey's Industrial Oil \& Fat Products 6 ${ }^{\underline{a}}$ ed. v.1, John Wiley \& Son, New York p.565-576, 2005.

SIMÕES, C. M. O; SCHENKEL, E. P; GOSMANN, G; MELLO, J. C. P; MENTZ, L. A; PETROVICK, P. R. Farmacognosia: da planta ao medicamento. Porto Alegre/ Florianópolis: ed. UFRGS/ ed. UFSC, 1999.

SOUZA, S. A. M.; MEIRA, M. R.; FIGUEIREDO, L. S.; RONIE, M. E.; Óleos Essenciais: Aspectos Econômicos e Sustentáveis. Enciclopédia Biosfera, V.6, n.10, p.1-11, 2010.

SOUZA FILHO, O. C. de; SAGRILLO, M. R; GARCIA, L. F. M; MACHADO, A. K; CADONÁ, F; RIBEIRO, E. E; DUARTE, M. M. M. F; MOREL, A. F; CRUZ, I. B. M. da. The In Vitro Genotoxic Effect of Tucuma (Astrocaryum aculeatum), an Amazonian Fruit Rich in Carotenoids, Journal of Medicinal Food. November, v.16, n.11, p.1013-1021, 2013.

SUFRAMA. Superintendência da Zona Franca de Manaus. Disponível em < http://www.suframa.gov.br/invest/zona-franca-de-manaus-amazonia-ocidental.cfm> Acessado em 17 de Agosto 2015.

TINOCO, A. C. Açaí amazônico: novas perspectivas de negócio. Belém, PA: Embrapa Amazônia Oriental, 2005. 1 CD-ROM. Trabalho apresentado no Workshop Regional do Açaizeiro: pesquisa, produção e comercialização, Belém, PA, 2005.

VILAPLANA, A. G-; BAENAS, N; VILLAÑO, D; SPEISKY, H., GARCÍA-VIGUERA, C; MORENO, D. A. Evaluation of Latin-American fruits rich in phytochemicals with biological effects Journal of Functional Foods, v.7, [s. n.], p.599-608, 2014.

WEBOFSCIENCE, 2015. <http://www. webofknowledge.com>. Acessado em 10 agosto de 2015.

YUYAMA, L. K. O. , AGUIAR, J. P. L. , FILHO, D. F. S., YUYAMA, K. , VAREJÃO, M. J., FÁVARO, D. I. T., VASCONCELLOS, M. B. A., PIMENTEL, S. A., CARUSO, M. S. F., Caracterização físico-química do suco de açaí de Euterpe precatoria Mart. oriundo de diferentes ecossistemas amazônicos. Acta Amazonica, v.41, n.4, p.545 $-552,2011$. 